\title{
Partial nephrectomy versus thermal ablation for clinical T1 renal tumours
}

\author{
Tobias Klatte ${ }^{1,2}$, Grant D. Stewart ${ }^{2,3}$ \\ ${ }^{1}$ Department of Urology, Royal Bournemouth Hospital, Bournemouth, UK; ${ }^{2}$ Department of Surgery, University of Cambridge, Cambridge, UK; \\ ${ }^{3}$ Department of Urology, Cambridge University Hospitals, Cambridge, UK \\ Correspondence to: Tobias Klatte, MD, FEBU, FRCSEd. Department of Urology, Royal Bournemouth Hospital, Castle Lane East, Bournemouth, \\ BH7 7DW, UK. Email: tobias.klatte@gmx.de. \\ Provenance: This is an invited article commissioned by the Section Editor Dr. Xiao Li (Department of Urology, Jiangsu Cancer Hospital, Jiangsu \\ Institute of Cancer Research, Nanjing Medical University Affiliated Cancer Hospital, Nanjing, China). \\ Comment on: Andrews JR, Atwell T, Schmit G, et al. Oncologic Outcomes Following Partial Nephrectomy and Percutaneous Ablation for cT1 Renal \\ Masses. Eur Urol 2019;76:244-51.
}

Submitted Aug 23, 2019. Accepted for publication Aug 30, 2019.

doi: 10.21037/atm.2019.09.12

View this article at: http://dx.doi.org/10.21037/atm.2019.09.12

The incidence of renal tumours has been increasing over the last decades because of more frequent abdominal imaging, leading to higher numbers of renal tumours identified at an early stage (1). Guidelines emphasise surgery as a preferred treatment modality for patients with clinical T1N0M0 tumours, but consider thermal ablation and active surveillance as an alternative approach for T1a lesions $(2,3)$. High-quality studies comparing partial nephrectomy $(\mathrm{PN})$ and thermal ablation are sparse, and the majority of studies are limited by small numbers, short follow-up and their retrospective design.

A study by Andrews et al. (4) published in European Urology aimed to provide more evidence on management strategies for the clinical T1N0M0 renal tumour. The authors reported on oncological outcomes of 1798 patients, who underwent $\mathrm{PN}$ or percutaneous thermal ablation [radiofrequency ablation (RFA) or cryoablation (CA)] at the Mayo Clinic in Rochester, United States, between 2000 and 2011. Outcomes were stratified according to clinical T1a (confined to the kidney, $\leq 4 \mathrm{~cm}$ ) and clinical T1b (confined to the kidney, $4.1-7 \mathrm{~cm}$ ). All patients were first evaluated in the Department of Urology, and those interested in thermal ablation or deemed to be unfit for surgery were referred to interventional radiology for discussion of thermal ablation. After a median follow-up of 6.0 to 9.4 years, there were no statistically significant differences in local recurrencefree survival, metastasis-free survival and cancer-specific survival between PN and thermal ablation for both T1a and T1b tumours. Patients treated with thermal ablation had greater all-cause mortality, because older and comorbid patients with limited life expectancy had thermal ablation rather than PN. This study provides evidence that, with appropriate patient selection and treatment at a highvolume tertiary care centre, excellent long-term outcomes can be achieved by both PN and thermal ablation. The study did not comment on renal functional outcomes or complications.

Despite the large number of patients studied, conclusions might only be drawn for clinical T1a tumours. As per local policy at the Mayo Clinic, patients with T1b tumours underwent CA rather than RFA if thermal ablation was contemplated. The numbers of patients undergoing CA for T1b tumours was fairly small $(\mathrm{n}=52)$, which limits conclusions in this subgroup. There was no difference in local recurrence-free survival after CA and PN, but metastasis-free survival and cancer-specific survival appeared to be worse after CA despite absence of statistical significance. In contrast, data from the Cleveland Clinic showed that CA conveyed worse local recurrence-free survival than $\mathrm{PN}$ in clinical T1b tumours [estimated 5-year probability: $70 \%$ vs. $100 \%$, (5)]. These data appear to be in line with RFA outcomes reported by Psutka et al. (6), although definitions of recurrence varied across studies. In view of the limited evidence on thermal ablation, it must be 
concluded that surgery remains the mainstay of therapy for T1b tumours, but thermal ablation may be considered as an option for highly selected patients.

Among the "small" (i.e., clinical T1a) tumours analysed, there was significant selection bias. The authors were not able to account for anatomical scoring systems such as the PADUA or the RENAL nephrometry score (7), but it is fairly evident that there were significant differences between groups. RFA was generally performed in patients with peripheral, posterior tumours $\leq 3 \mathrm{~cm}$ which showed no proximity to the ureter. It is therefore highly likely that the majority of RFA patients had low complexity T1a lesions, in contrast to those undergoing $\mathrm{PN}$ or CA. The relatively low 5-year metastasis-free survival probability of $93.9 \%$ for RFA (compared with $98.0 \%$ for PN and $100 \%$ for CA) should therefore be seen with a cautious eye. As increasing tumour complexity correlates with adverse pathological features such as higher grades (8), the relative difference in metastasis-free survival between RFA and PN/CA may actually increase if one would adjust for a nephrometry score.

A significant proportion of patients did not have a renal tumour biopsy prior to thermal ablation, especially prior to RFA. Among those with available histology, between 21\% $(\mathrm{PN})$ and $35 \%(\mathrm{CA})$ had treatment for a benign T1a renal tumour. At many centres, the histology is only obtained at the time of $\mathrm{PN}$ or thermal ablation, and has virtually zero impact on management decisions. In contrast, uncoupling a renal tumour biopsy from actual treatment benefits patient counselling and might avoid overtreatment of benign tumours. Indeed, Tanabalan et al. (9) reported the outcomes of a prospective study from a high volume kidney cancer specialist centre, in which all patients with cT1a renal tumours were offered a biopsy over a 2 -year period. Among the 266 patients who underwent a biopsy, 78 (29\%) were found to have benign pathology. Seventy-five of the 78 patients $(96 \%)$ chose no intervention, which corresponds to $28 \%$ of all patients with cT1a tumours. However, treatment and thus potentially overtreatment of benign renal tumours is still common, and morbidity from these interventions is not negligible. In a nationwide study of 1,202 patients with surgically treated oncocytoma, $20 \%$ had in-hospital complications and 5 patients died within 60 days after surgery (10).

Another important limitation of this study is that it does not include patients managed by active surveillance, which is an accepted management strategy for small renal tumours. A meta-analysis of 8 uncontrolled studies showed excellent short- to intermediate-term metastasis-free and cancer-specific survival outcomes (11), comparable to the outcomes reported by Andrews et al. after intervention (4). Further, data from the prospective Delayed Intervention and Surveillance for Small Renal Masses (DISSRM) registry suggest that active surveillance is not inferior to immediate intervention (12), and that there is little difference in patient reported quality of life (13). Active surveillance can be recommended to patients of advanced age and multiple comorbidities, or to those in which other conditions take priority at a given time. It is worth mentioning that observation of small renal tumours often represents watchful waiting rather than active surveillance.

The optimal management strategy for clinical T1 renal tumours can only be determined by prospective clinical trials. Unfortunately, the study by Andrews et al. (4) represents another retrospective case series. Case series are cheap and easy to accomplish, but provide the weakest evidence for assessing the efficacy of treatment (14). There are only a limited number of completed and reported prospective surgical trials, and retrospective case series and expert opinions provide most evidence for clinical practice guidelines. More than $20 \%$ of surgical trials are discontinued early, most commonly owing to poor recruitment (15), and trials on surgical management of renal tumours are no exception. Indeed, both SURAB (ISRCTN31161700) and CONSERVE (ISRCTN23852951) did not complete recruitment in the feasibility phase. Recently, a feasibility trial of a cohort embedded randomised controlled trial comparing Nephron Sparing Treatment (NEST) has started to recruit in the United Kingdom (ISRCTN18156881). Although NEST will mainly compare percutaneous CA with robotic $\mathrm{PN}$ in patients with biopsy-proven renal cell carcinoma, those patients screened but not invited for treatment will remain part of the trial cohort. As such, oncological outcomes, patient reported outcome measures and biomarkers will also be assessed in those not assigned to the treatment or control arm. The feasibility phase will be conducted at a single high-volume centre and is expected to progress to a full multicentre trial if recruitment is successful (16). The randomised Robot-assisted Surgical Resection vs. Cryoablation of Localised Renal Cancer (ROAST) trial will be launched in Denmark in September 2019 (NCT03390413). The primary outcome of this trial is change in renal function at 6 months, and secondary outcomes include incomplete treatment, complications and costs. Finally, the European Active Surveillance of Renal Cell Carcinoma (EASE) study is currently recruiting 
patients with biopsy proven renal cell carcinoma $\leq 4 \mathrm{~cm}$. The primary objective of this prospective registry is to evaluate overall survival. These trials will shed light on the optimal management of patients with early stage renal tumours.

\section{Acknowledgments}

None.

\section{Footnote}

Conflicts of Interest: GD Stewart has received educational grants from Pfizer, AstraZeneca and Intuitive Surgical, consultancy fees from Pfizer, Merck, EUSA Pharma and Cambridge Medical Robotics, travel expenses from Pfizer and speaker fees from Pfizer. These are not relevant to the content of this article. T Klatte has no conflicts of interest to declare.

Ethical Statement: The authors are accountable for all aspects of the work in ensuring that questions related to the accuracy or integrity of any part of the work are appropriately investigated and resolved.

\section{References}

1. Rossi SH, Klatte T, Usher-Smith J, et al. Epidemiology and screening for renal cancer. World J Urol 2018;36:1341-53.

2. Ljungberg B, Albiges L, Abu-Ghanem Y, et al. European Association of Urology Guidelines on Renal Cell Carcinoma: The 2019 Update. Eur Urol 2019;75:799-810.

3. Campbell S, Uzzo RG, Allaf ME, et al. Renal Mass and Localized Renal Cancer: AUA Guideline. J Urol 2017;198:520-9.

4. Andrews JR, Atwell T, Schmit G, et al. Oncologic Outcomes Following Partial Nephrectomy and Percutaneous Ablation for cT1 Renal Masses. Eur Urol 2019;76:244-51.

5. Caputo PA, Zargar H, Ramirez D, et al. Cryoablation versus Partial Nephrectomy for Clinical T1b Renal

Cite this article as: Klatte T, Stewart GD. Partial nephrectomy versus thermal ablation for clinical T1 renal tumours. Ann Transl Med 2019;7(Suppl 8):S363. doi: 10.21037/ atm.2019.09.12
Tumors: A Matched Group Comparative Analysis. Eur Urol 2017;71:111-7.

6. Psutka SP, Feldman AS, McDougal WS, et al. Long-term oncologic outcomes after radiofrequency ablation for T1 renal cell carcinoma. Eur Urol 2013;63:486-92.

7. Klatte T, Ficarra V, Gratzke C, et al. A Literature Review of Renal Surgical Anatomy and Surgical Strategies for Partial Nephrectomy. Eur Urol 2015;68:980-92.

8. Kutikov A, Smaldone MC, Egleston BL, et al. Anatomic features of enhancing renal masses predict malignant and high-grade pathology: a preoperative nomogram using the RENAL Nephrometry score. Eur Urol 2011;60:241-8.

9. Tanabalan C, Tadtayev S, Raman A, et al. Routine use of biopsy in the management of the small renal mass. J Clin Urol 2017;10:abstr 58.

10. Neves JB, Withington J, Fowler S, et al. Contemporary surgical management of renal oncocytoma: a nation's outcome. BJU Int 2018;121:893-9.

11. Pierorazio PM, Johnson MH, Patel HD, et al. Management of Renal Masses and Localized Renal Cancer: Systematic Review and Meta-Analysis. J Urol 2016;196:989-99.

12. Pierorazio PM, Johnson MH, Ball MW, et al. Five-year analysis of a multi-institutional prospective clinical trial of delayed intervention and surveillance for small renal masses: the DISSRM registry. Eur Urol 2015;68:408-15.

13. Patel HD, Riffon MF, Joice GA, et al. A Prospective, Comparative Study of Quality of Life among Patients with Small Renal Masses Choosing Active Surveillance and Primary Intervention. J Urol 2016;196:1356-62.

14. Horton R. Surgical research or comic opera: questions, but few answers. Lancet 1996;347:984-5.

15. Chapman SJ, Shelton B, Mahmood H, et al. Discontinuation and non-publication of surgical randomised controlled trials: observational study. BMJ 014;349:g6870.

16. Neves JB, Cullen D, Grant L, et al. Protocol for a feasibility study of a cohort embedded randomised controlled trial comparing NEphron Sparing Treatment (NEST) for small renal masses. BMJ Open 2019;9:e030965. 